Investigations

\title{
Diabetes Group Visit in a Primary Care Setting
}

\author{
Selma Mujezinovic Rochester \\ Nursing, Long Pond Medical Group, USA
}

Article history

Received: 06-07-2016

Revised: 04-08-2016

Accepted: 08-09-2016

Email: mselmabsn@hotmail.com

\begin{abstract}
The group visits models one innovation in the field of chronic illness management that shows promise in meeting the growing demands for this type of care. Patients get significant peer and healthcare provider support during group visits. Group visits typically last $90 \mathrm{~min}$ and occur on a regularly scheduled basis. The study design was a quasi-experimental prepost-test with a comparison group. Patients were recruited into either the intervention or control group. Patients self-selected which group they chose to participate in. The measurement data was collected at three time intervals: baseline, 3-months and 6-months. The control group received standard of care. Changes in clinical indicators and in patient self-efficacy were tested using Repeated Measures ANOVA. The intervention group receiving diabetes group visits showed statistically significant improvement in all variables with the exception of diastolic blood pressure. In order to achieve improved patient outcomes and reduce the socioeconomic burden of diabetes care, the group care model should be implemented as a standard of care in diabetes management.
\end{abstract}

Keywords: Diabetes Group Visits, Shared Medical Appointment, Advanced Practice Nurse (APN) Lead Group Visits, Diabetes Mellitus

\section{Introduction}

The Centers for Disease Control and Prevention (CDC) predicts a dramatic increase in diabetes between 2010 and 2025 (CDC, 2015) and the Institute for Alternative Futures diabetes model estimates that the number of Americans living with diabetes (diagnosed and undiagnosed) will increase $64 \%$ by 2025 from $32,300,000$ to $53,100,000$ people. The resulting medical and societal cost of diabetes will be up to $\$ 514.4$ billion by 2025 , a $72 \%$ increase from 2010 (CDC, 2015). A diagnosis of diabetes can require multiple changes in a person's behavior, diet and lifestyle. Efforts to sustain these changes and manage this complex chronic disease can be difficult. Group visits, in which several patients meet together with a primary care provider and interdisciplinary team, have tremendous potential to improve health care quality, cost and access. When group based diabetes self-management education and a primary care visit occur within a single appointment, people with the disease can address multiple needs in one visit and take advantage of peer groups for support and motivation. Healthy People 2020 and the American Diabetes Association outline (ADA, 2015) several objectives to improve the quality of life and reduce the disease burden for all people with diabetes.
To achieve these goals, they recommend three key components for effective disease management planning: regular medical care, self-management education and ongoing diabetes support.

Diabetes mellitus is a dangerous chronic disease that can lead to cardiovascular complications, renal disease, damage to the retina causing blindness and nerve damage causing neuropathy (Reitz et al., 2012). Diabetes places a significant financial burden on the health care system. On average, people with diabetes accrue 2.3 times more cost than healthy individuals due to complications of the disease (Reitz et al., 2012). Diabetes mellitus causes patients significant disability which leads to decreased quality of life. The needs of diabetic patients do not only include adequate glycemic control but also preventing complications, disability limitations and rehabilitation. There are seven essential self-care behaviors that predict good outcomes in diabetics: healthy eating, being physically active, monitoring of blood sugar, compliance with medications, good problem-solving skills, healthy coping skills and risk-reduction behaviors (Reitz et al., 2012). These seven behaviors have been found to be positively correlated with good glycemic control, reduction of complications and improvement in quality of life. Individuals with diabetes have been shown to make a dramatic impact on the progression and development of their disease by 
participating in their own care. The incidence and prevalence of Type 2 Diabetes Mellitus (T2DM) have reached epidemic proportions in the United States. Early initiation of treatment can prevent or delay disease progression and reduce the risk for diabetes-related complications. The goal is to achieve evidence-based clinical goals through implementation of effective management strategies that substantially reduce the risk of morbidity and mortality and, ultimately, improve patient outcomes (Deakin et al., 2005).

\section{Review of Literature}

A review of the literature regarding diabetes group visits provided strong evidence of the effectiveness of these type of visits. Group visits include most of the components of individual visits, usually including private or semiprivate one-on-one evaluations conducted by a healthcare provider at each visit, as well as group educational sessions that emphasize patient selfmanagement and address topics such as medical and pharmaceutical management, nutrition, exercise and psychosocial contributors to health and illness (Jaber et al., 2006). In a study conducted by Jaber et al. (2006), participation in the group visit program was associated with reaching the goals of having a blood pressure less than $140 / 90 \mathrm{mmHg}$, a significant decline in calculated LDL and a hemoglobin A1C concentration less than 7\%. Group visits can improve the quality of chronic disease management compared to the usual office visit. They provide more time for self-management education and skill building and may reduce perceived barriers to behavior change. Group visits can improve patient satisfaction by increasing patients' trust in providers and promoting patients' engagement in their care. Studies have demonstrated group visit programs can reduce emergency department visits, visits to specialists and hospital admissions and the associated costs of care (Reitz et al., 2012). Diabetes Self-Management Education (DSME) has been shown to be most effective when delivered by a multidisciplinary team with a comprehensive plan of care. Generally, the literature favors current practice that utilizes the registered nurse or Advanced Practice Nurse (APN), registered dietitian and the registered pharmacist as the key primary instructors for diabetes education (Reitz et al., 2012). This should occur in collaboration with members of the multidisciplinary team who are responsible for designing the curriculum and assisting in the delivery of DSME.

Shared medical appointment visit time is much longer (60-90 $\mathrm{min})$ than what is spent with each patient at a typical office visit (15-20 min). Group visits seem particularly suited to chronic illness management in that they allow more time for self-management education, skill-building and healthcare provider-patient interaction. Group education reinforces messages received in the individual office visit, increases perceived benefits and provides social persuasion and effective action cues (Deakin et al., 2005). Group visits reinforce patients' self-efficacy which is strongly associated with successful chronic disease self-management modeling (Deakin et al., 2005). Additionally, seeing others that have accomplished the desired behavior and overcome obstacles is another powerful contributor to patient self-efficacy. The change in delivery of care via group visits improves patient and healthcare provider satisfaction, quality of care, quality of life, as well as, decreased health care utilization, particularly visits to the emergency department and specialists, rates of hospitalization or readmission (Deakin et al., 2005). Shared visits are a promising approach to chronic care management for the motivated patient. They provide a mechanism for providing time for education combined with clinical care in a manner that at least maintains productivity and revenue (Deakin et al., 2005). The combination of individual clinical attention and group education, if well-designed, has the potential to address multiple aspects of patient care in a personalized, tailored fashion but may only be applicable to motivated patients who are willing to invest extra time in their care. Thus, the group visit concept is a useful addition to the chronic care model (Deakin et al., 2005).

\section{Purpose of the Study}

The purpose of this study was to evaluate whether group visits delivered as routine diabetes care and structured according to a systematic education approach, were more effective than individual appointments in improving metabolic control as evidenced by reduction of Hemoglobin A1C (A1C), Low Density Lipoprotein (LDL), Blood Pressure (BP) and improved self -efficacy in adult patients with type 2 diabetes.

\section{Research Questions}

- Is there an improvement in self-efficacy from baseline to 6 months between T2DM patients who participate in diabetes group visits and T2DM patients who receive standard care?

- Are there differences in self-efficacy between T2DM patients who participate in diabetes group visits compared to T2DM patients who receive standard care?

- Are T2DM patients who participate in diabetes group visits more successful at improving A1C, LDL and BP as compared to T2DM patients who receive standard care?

\section{Method and Design}

The present study took place in a primary care site in a suburban clinic located in Western New York. Institutional Review Board (IRB) approval was received 
from two participating institutions and the study was conducted following IRB guidelines in order to ensure human subjects protection. All participants showed their initial consent to participate in the study by willingly contacting the Primary Investigator (PI) to be a part of the study. At the beginning of the first group visit, the PI explained the study and requested completion of the consent form. All participants then signed a written consent form prior to participation in the study and all forms were placed in an envelope to maintain confidentiality of the subjects. Patients were given the contact information for the PI in case they had any further questions. At the first session, any patient who chose not to complete the consent form was allowed to stay for the group visit if desired. When those cases arose, no data was collected from those patients. The PI collected measurement data at three time intervals: baseline, 3-month assessment and 6-month assessment.

Patients with the diagnosis of T2DMwho met the study criteria who were identified by their Primary Care Provider (PCP) were referred to the PI. The inclusion criteria were: A1C $6 \%$ or higher, baseline blood work of LDL available, BP readings at baseline available, adults over 18 years of age, male or female and English speaking. The exclusion criteria were pediatric patients, patients with Type 1 Diabetes Mellitus, pregnant patients with gestational diabetes and non- English speaking patients. The PI sent a letter describing the study to each patient. The same letter was handed to the patient to invite them to participate in the study during their routine medical care appointment after the patient and their Primary Care Provider (PCP) had the opportunity to discuss the diabetes group visits. Patients who agreed to participate self-selected into the control or intervention group.

The intervention which consisted of six 90 min group educational sessions began after baseline data collection. The intervention group participated in the sessions, while the comparison group received the normal standard of care. The standard of care consisted of office visits with the PCP during which lab data was reviewed and medication dosing changes were made, if needed. Due to the time constraints and multiple medical issues addressed during usual care appointments, limited education was provided to the patient. The usual care visit provided basic glucose monitoring information, basic nutrition counseling and medication readjustment. The PI obtained the same measures from both of the groups including A1C, LDL and BP at baseline, 3 and 6 -months. The self-efficacy tool was administered at baseline and at 6 months. These were the dependent variables in this study. The PI had 2 afternoon sessions blocked per month to lead the diabetes group visit during that timeframe. Family members of patients participating in the group visit were invited to attend with their loved one.
A registered dietician facilitated the nutrition lecture and a clinical pharmacist facilitated the instruction on medication management with the PI who was an APN. The curriculum was developed by PI with guidance of content experts in the community. The written curriculum reflecting current evidence and practice guidelines was provided to patients. The curriculum covered diabetes-related complications such as cardiovascular complications including myocardial infarction and cerebrovascular accident, peripheral vascular disease and diabetic neuropathy, renal disease and diabetic nephropathy, diabetes related vision changes and diabetic retinopathy. The nutrition guidelines, carbohydrate counting, meal planning, simple menu options, diabetes medications including oral and inject able options were also included. Participants gained skills in choosing healthy food alternatives, blood glucose monitoring, medication management and benefits of exercise. Two groups of 10 participants met on a monthly basis for six months. Nursing staff took participant's vital signs and reviewed medications. The PI performed a one-on-one exam prior to the group visit. The whole group met for 90 min during which their blood work was reviewed. The participants received their report card at the beginning of the group visit, with plus indicating that they had met their goal, or a minus indicating that they were not at their goal. The PI then led the discussion and lecture. Participants were advised to ask questions throughout the presentation. Participants were reminded that they could refuse to participate without compromising their medical care at any time during the group sessions. All participants showed their initial consent to participate in the study by willingly contacting the PI to be a part of the study. At the beginning of the first group visit, the PI explained the study and requested completion of the consent form.

Systematic education during the group visits was provided in accordance to American Diabetes Association (ADA) guidelines. The curriculum was developed by the PI, reviewed and approved by diabetes experts in the community and faculty members at a local nursing school. The PI was responsible for all education during the diabetes group visits; content experts did coteach nutrition and medication management. The Electronic Medical Record (EMR) was utilized to gather quantitative data of patients who participated in the study. The data that was collected from the EMR was A1C, LDL and BP readings. Demographic data were also collected and included age, race and gender. After the completion of three group visits, mid intervention data (A1C, LDL and BP) was collected. The same data was collected at the end of the study after 6 group visits. The data was collected and used from the patients who participated in three or more group visits. If the patient completed less than three group visits their data was not used. In addition to the three previous measures, all 
patients took a self-efficacy tool survey called the "Diabetes Self-Efficacy Scale" at the beginning and at the end of the study. The tool was free to use without permission (Lorig et al., 2005). The Diabetes SelfEfficacy Scale measured patients' confidence in management of their diabetes and provided another measurement that reflected effectiveness of group visits. In order to maintain confidentiality the PI removed anything containing names from the patient self-efficacy tool. The self-efficacy tool was coded with the patients' study number. This tool was mailed to the patients' home address along with a self-addressed stamped envelope to be returned to the PI. The PI used the EMR to access patients' lab results and blood pressure readings. The patients'A1C, LDL, SBP, DBP and self-efficacy survey tool was entered into SPSS Version 22.

\section{Results}

All variables were screened for normality and were found to be within acceptable limits. Changes in clinical indicators and in patient self-efficacy from baseline, 3 months and 6 months were tested using Repeated Measures ANOVA. For all tests, the assumption of sphericity was tested using Mauchly's Test of Sphericity. When the assumption was violated, the GreenhouseGeisser correction was used.

The intervention group receiving diabetes group visits showed statistically significant improvement in all variables with the exception of DBP (Table 1). Significant change was found in the A1C levels $(\mathrm{F}=$ 16.1, $\mathrm{p}<0.05)$. The change was such that A1C levels decreased from baseline $(\mathrm{M}=8.2, \mathrm{SD}=1.4)$ to three months measurement $(M=7.1, S D=0.88)$, to the six months measurement $(\mathrm{M}=6.7, \mathrm{SD}-0.64)$. The effect size was very strong (eta squared $=0.49$ ). The LDL results showed significant change $(\mathrm{F}=4.1, \mathrm{p}<0.05)$. The change was such that LDL decreased from baseline $(\mathrm{M}=86.4$, $\mathrm{SD}=25.4)$, to the three months $(\mathrm{M}=74.9, \mathrm{SD}=23.9)$, to the six months where it maintained lower value $(\mathrm{M}=$ 74.2, $\mathrm{SD}=17.8$ ). The effect size was moderate (eta squared $=0.20)$. The results of SBP analysis showed statistically significant change $(F=6.3, p<0.05)$. The change was such that SBP decreased from baseline $(\mathrm{M}=$ 133.3, $\mathrm{SD}=10.3)$, to the three months $(\mathrm{M}=126.4, \mathrm{SD}=$ $9.1)$, to six months where remained at lower value $(\mathrm{M}=$ 124.4, SD = 9.3). The effect size was moderately strong (eta squared $=0.26)$. The DBP did not significantly change in the intervention group $(F=0.79, p>0.05)$. The biggest impact was found on patient self-efficacy results. There was significant change in self-efficacy $(\mathrm{F}=78.7$, $p<0.05)$. The change was such that self-efficacy increased from the baseline $(\mathrm{M}=7.4, \mathrm{SD}=1.2)$ to the post measure $(\mathrm{M}=8.9, \mathrm{SD}=0.6)$. The effect size was very strong (eta squared $=0.81$ ).

In contrast, the control group only demonstrated significant change in patient self-efficacy; no clinical measures reached significant changes. There was no significant difference from pre to post analysis on A1C levels for the control group $(F=0.07, \mathrm{p}>0.05)$. The same test was used to assess change in LDL levels for the control group; again Greenhouse Geisser correction was used. There was no significant difference between pre to post analysis on LDL levels $(\mathrm{F}=2.8, \mathrm{p}>0.05)$. During the analysis of SBP for the control group because Mauchly's Test of Sphericity was not significant, no correction was made. The results revealed no significant difference in SBP pre to post analysis $(\mathrm{F}=$ $0.93, \mathrm{p}>0.05)$. The impact on DBP with standard of care was measured using the same test, there was no correction made as Mauchly's Test of Sphericity was not significant. The results showed no significant change from pre to post analysis $(\mathrm{F}=2.3, \mathrm{p}>0.05)$. The self-efficacy in patient receiving standard of care did show significant change from pre to post $(\mathrm{F}=7.8$, $p<0.05)$. The change was such that mean efficacy increased from baseline $(\mathrm{M}=7.2, \mathrm{SD}=1.4)$ to post measure $(\mathrm{M}=7.4, \mathrm{SD}=1.5)$, the effect size was moderate at (eta squared $=0.27$ ). Although this change was statistically significant, the effect size was notably weaker than in the intervention group.

Table 1. Diabetes group visit vs. standard of care

\begin{tabular}{|c|c|c|c|c|c|c|c|}
\hline \multirow[b]{2}{*}{ Test } & \multicolumn{2}{|c|}{ Baseline } & \multicolumn{2}{|c|}{3 Months } & \multicolumn{2}{|c|}{6 Months } & \multirow[b]{2}{*}{ Effect size eta squared } \\
\hline & $\mathrm{M}$ & SD & M & SD & M & SD & \\
\hline \multicolumn{8}{|l|}{ Control } \\
\hline $\mathrm{A} 1 \mathrm{C}$ & 7.9 & 2.2 & 8.0 & 2.20 & 8.0 & 2.3 & 0.000 \\
\hline LDL & 96.3 & 27.6 & 84.8 & 31.02 & 83.2 & 27.6 & 0.120 \\
\hline SBP & 133.4 & 12.9 & 128.6 & 12.50 & 128.2 & 15.7 & 0.040 \\
\hline DBP & 78.7 & 10.1 & 76.9 & 9.40 & 74.6 & 9.8 & 0.100 \\
\hline Self-efficacy & 7.2 & 1.4 & & & 7.4 & 1.5 & $0.27 *$ \\
\hline \multicolumn{8}{|l|}{ Intervention } \\
\hline A1C & 8.2 & 1.4 & 7.1 & 0.9 & 6.9 & 0.6 & $0.49 *$ \\
\hline LDL & 86.4 & 25.4 & 74.8 & 23.9 & 74.3 & 17.8 & $0.20 *$ \\
\hline SPD & 133.3 & 10.3 & 126.4 & 9.1 & 124.4 & 9.3 & $0.26^{*}$ \\
\hline DBP & 77.1 & 8.7 & 75.1 & 7.3 & 74.5 & 8.5 & 0.040 \\
\hline Self-efficacy & 7.4 & 1.2 & & & 8.9 & 0.6 & $0.81 *$ \\
\hline
\end{tabular}

$* \mathrm{p}<0.05$ 


\section{Discussion}

This study revealed significant positive differences between group visit (intervention) and control patients in adherence to ADA standards and self -reported diabetes self-efficacy. Perhaps the longer duration of group visits provides more time to address guidelines than a typical primary care encounter. Additionally, the PI was able deliver consistent messages to multiple patients simultaneously in group visits, rather than repeating them individually to multiple patients. Monthly appointments provide more frequent contact with the healthcare provider increasing opportunities to systematically address ADA guidelines.

The discussion of individual patient issues in groups may have contributed to other patients' accepting referrals and tests, from diminished anxiety and a desire to show other group members' commitment to their own health care. With open general discussions in group visits, patients educated each other about their experience; perhaps hearing information from their peers resulted in higher acceptance of suggestions from the PI.

Patients' financial limitations might have led to significant differences in A1C, LDL, or BP control, by prohibiting purchase of prescribed medications or healthier food, thus creating a ceiling effect for benefits of improved guideline adherence. Clinical outcomes were under the patients' control, depending on them following lifestyle guidelines and adhering to medication regimens. Incorporating motivational and behavioral strategies emphasizing patients' daily responsibilities and skill building for healthy lifestyles compatible with diabetes seems to affect improvements in clinical outcomes. The intervention group receiving diabetes group visits showed statistically significant improvement in all variables with the exception of DBP. The selfefficacy in patients receiving standard of care did show positive change from pre-to-posttest. Although, this change was statistically significant, the effect size was notably weaker than in the intervention group.

We all recognize that the U.S. health care system suffers from serious gaps in quality and widespread waste has stimulated a broad array of public and privates sector initiatives to improve performance. These include not only public reporting, pay for performance and quality improvement programs but also major initiatives by the organizations responsible for institutional accreditation and professional certification. The underlying goal of these efforts is to improve the quality and lower the cost of care by fostering greater accountability on the part of providers for their performance.

Chronic illness is proving to be one of the most pressing public health issues of the 21 st century. Managing chronic diseases, which includes gaining a comprehensive knowledge of patients and addressing their risky behaviors, can be complicated by current demands on health care provider time. The focus in on patient centered care that eliminates access barriers and improves quality, outcomes and practice finances. Group visits, which were identified as an important facet of a "new model" of care, allow health care provider to deliver extensive patient education and self-management instruction while possibly increasing financial productivity. It is estimated that group visits have the potential of generating an additional $\$ 15,411$ per health care provider per year (Jaber et al., 2006). In addition, group visits offer patients with similar illnesses an opportunity to interact with and learn from one another.

Topics, such as medication management, stress management, exercise and nutrition and community resources, may be suggested by the group facilitator or by patients, who raise concerns, share information and ask questions. In programs emphasizing selfmanagement, the health care provider and patients work together to create behavior change action plans, which detail achievable and behavior specific goals that participants aim to accomplish by the next session, for example improved compliance with blood sugar readings, food diary etc. Once plans are set, the group discusses ways to overcome potential obstacles, which raises patients' self-efficacy and commitment to behavioral change. Patients' family members can also be included in these group sessions.

In 1995, Harvard business school professors Clayton Christensen and Joseph Bower put "disruptive technologies" in the business lexicon by introducing the term in a seminal article in the school's journal. Patients embrace innovations because they represent value savings, convenience, access, quality, or a combination of these. Diabetes group visits have been proven as the effective strategy to improving patients' outcomes, reducing comorbid diseases, preventing costly surgery resulting in diabetes complications. The diabetes group visits are a form of disruptive innovation. As the overall emphasis on reducing cost continues, diabetes group visits will hopefully become standard of diabetes care along with individualized visits. They provide education regarding diabetes management, focusing on diabetes complication prevention and improving self-efficacy. The healthcare providers in the primary care setting see up to 15 patients with diabetes per day. They repeat same the instructions to all 15 patients day after day. The idea of placing these same patients in a classroom and providing them education related to disease management strategies, blood sugar monitoring, disease complications and medication management was a novel but worthwhile pursuit.

\section{Study limitations}

This study was small with 20 patients in the intervention group and 22 patients in the control group. The practice setting was a suburban medical practice which serves a very homogenous population of mostly white patients. Both the small sample size 
and the practice setting limit the ability to generalize the results to the population at large. Additionally, this was a nonrandomized study; therefore patients who were more motivated to change may have been more likely to participate in the groups and had more favorable results. Patients who have diabetes are frequently seen every three months; any improvement in their data could be correlated to monthly provider visits that were required to participate in the study. Finally, the study was short in duration. The question is whether these improvements could be sustained long term.

\section{Implications for Practice}

Despite the study limitations, the feasibility of implementing effective diabetes group visits using a group interdisciplinary team approach to care for motivated patients with diabetes was demonstrated. The significant changes and effect sizes, compared with those patients who received standard care, indicated that shared medical appointments for diabetes constitute a practical system redesign that may improve patient outcomes. The group visit model of care is a primary care system change designed to overcome the challenges of the traditional 15 to 20 min visit and underused selfmanagement education. This interdisciplinary model of care offers peer support and motivation that can help participants better cope with and manage their diabetes through the shared life experiences of group members (Burke and O'Grady, 2012). The peer interactions are instrumental in facilitating positive lifestyle and behavior changes by creating a supportive clinical and social environment. In addition, group visits shift the responsibility away from the provider as an expert imparting knowledge, to the patient and peer group members, with each member taking an active role in the process. Patients with diabetes are known to be at increased risk of cardiovascular disease and cardiovascular related death. There was a statistically significant reduction in systolic but not in the diastolic blood pressure among patients attending diabetes group visits. Many lifestyle modifications such as weight reduction, dietary changes, physical activity and alcohol consumption have been found to reduce systolic blood pressure. All of these risk factors were discussed during the group visits. During the group visits the APN used the inter-professional approach that allowed other healthcare professionals to address health behaviors, emotional and physiological aspects of care. Each team member contributed their expertise. The group visits provided greater interaction and support of other patients. In the group, patients share their personal experiences. The patients encouraged each other and gave useful recommendations on how to solve barriers to their care. Patients may have similar questions and learn from each other. Shared medical appointments offer greater patient education and the educational information does not need to be repeated, as it would for an individual patient visit. Patient education is geared toward self-care and lifestyle interventions. There was more time to address patients' inquiries and concerns during group visits. Primary care practices are ideal locations to implement diabetes group visits; the patients can be educated about the diabetes disease process, disease complications, disease management and lifestyle changes that will slow the disease progression. Group visits can provide a new sustainable model for diabetes education and management. APN facilitated group visits are an innovation in healthcare that can improve both access to care and health outcomes. Involving patients in their own care leads to improved self-efficacy, empowerment and can increase efficiency in the healthcare system.

\section{Ethics}

This article is original and contains unpublished material. The corresponding author confirms that all of the other authors have read and approved the manuscript and no ethical issues involved.

\section{References}

ADA, 2015. Strategies for improving care. Sec. 1. In Standards of Medical Care in Diabetes-2015. Diabetes Care, 38: S5-S7.

Burke, R.E. and E.T. O'grady, 2012. Group visits hold great potential for improving diabetes care and outcomes But Best Practices Must Be Developed. Health Affairs, 31: 103-109. DOI: $10.1377 /$ hlthaff.2011.0913

CDC, 2015. Center for disease control.

Deakin, T.A., C.E. Mcshane, J.E. Cade and R. Williams, 2005. Group based training for selfmanagement strategies in people with type 2 diabetes mellitus. Cochrane Database Systematic Rev. DOI: 10.1002/14651858.cd003417.pub2

Jaber, R., A. Braksmajer and J. Trilling, 2006. Group visits: A qualitative review of current research. J. Am. Board Family Medicine, 276-290.

Lorig, K.R., 2005. Outcomes of border health spanish/english chronic disease self-management programs. Diabetes Educator, 31: 401-409. DOI: $10.1177 / 0145721705276574$

Reitz, J.A., M. Sarfaty, J.J. Diamond and B. Salzman, 2012. The effects of a group visit program on outcomes of diabetes care in an urban family practice. J. Urban Health, 89: 709-716. DOI: $10.1007 / \mathrm{s} 11524-012-9675-9$ 